\title{
Japan heats up rice sequencing
}

Prompted by a looming threat from the commercial sector, Japan has brought forward the deadline of the International Rice Genome Sequencing Project (IRGSP) by almost four years to 2004. Although Japan plans to inject additional funding into the project to speed up sequencing, some think administration changes are also needed to accelerate the project.

Led by Japan, IRGSP is an 11-nation effort to sequence the entire 430-megabase rice genome. Members of the project, which began in 1998, currently include USA, EU, India, China, South Korea, Singapore, Thailand, and Canada.

Japan's Ministry of Agriculture, Forestry and Fisheries (MAFF; Tokyo), which oversees the Japanese contribution, asked for a total of $¥ 7.4$ billion (US $\$ 67$ million) for rice genome research in its budget proposal for the fiscal year 2000, a threefold increase from 1999. Of this, $40 \%$ will be allocated toward sequencing of the rice genome.

Rice genome-related research has been promised greater support thanks to Prime Minister Keizo Obuchi's Millennium Project, which aims to promote industries in areas such as biotechnology, information sciences, and the environment. This encompasses not only rice genome sequencing, but also a new interministerial project to create a library of full-length rice cDNA, as well as functional genomics and proteomics projects for agronomical use.

According to Takuji Sasaki, leader of MAFF's Rice Genome Research Program, the increased funding will overcome the main limiting factor in the sequencing effortcost. Although the program will now be able to sequence faster, there will be no changes to its initial phase. "We will keep to our plan to map ESTs of chromosomes 1, 5, and 6, and pursue the sequencing of 1 and 6 [allocated to Japan]," he says.

The decision to accelerate the sequencing effort was triggered by intensifying competition in plant genome research from the commercial sector, according to Ryu Ohsugi, director of MAFF's genome research office. Celera Genomics (Rockville, $\mathrm{MD}$ ), for example, is planning to sequence the rice genome, although it says it is likely to use indaco rice; IRGSP is focusing on a single cultivar of japonica variety rice (Nature 401, 520).

IRGSP researchers agree. "I am sure there are successful commercial efforts in rice

Asako Saegusa is a freelance writer working in Tokyo, Japan. genome research," says Ben Burr, a plant biologist from the Brookhaven National Laboratory (NY), "But rather than being detrimental to the public effort, such competitions have actually helped strengthen support for the project."

Nevertheless, some think the Japanese effort would proceed even faster if MAFF allowed institutes from other government ministries to become involved in the project. "Rice genome-related research has been dominated by MAFF, and unrelated institutes have never really had the chance

\section{The Japanese effort would proceed even faster if MAFF allowed institutes from other government ministries to become involved in the project.}

to become involved with such research," says one director of a leading genome institute. "We've always been happy to help out with the sequencing, but MAFF has never asked."

Kozo Murakami, emeritus professor in applied molecular biology from Nagoya University (Nagoya), agrees. "Future rice genome research should be carried out on a national level by involving other sciencerelated ministries," he says, "We've already done this with SNPs research; I don't see why it wouldn't work with rice, given its commercial potential and contribution to future plant research."

Meanwhile, in October, the US government awarded \$12.3 million to the US component of the project. The Institute for Genomic Research (TIGR; Rockville, MD) and a consortium of universities-Clemson University (SC), Cold Spring Harbor Laboratory (NY), and Washington University in St. Louis (MO) - will now sequence 50 megabases of the rice genome in three years, beginning with the 24-megabase chromosome 10, which they aim to complete by 2001 .

And European researchers expect to receive funding next year to begin sequencing their allocation-chromosome 2. Mike Bevan, professor in molecular genetics at the John Innes Centre (Norwich, UK), says they are currently considering a clone-by-clone approach supplemented by whole-genome shotgun sequencing "to try and complete the job in less than two years.”

Asako Saegusa 